\title{
Discovery
}

\section{Aspergillosis, Biological and the Prospective Impact on a Worldwide}

\author{
Retina \\ Department of Agricultural Research and Extension Center in USA
}

\begin{abstract}
The original definition of Biological started out as aset of preventive measures designed to reduce the risk of transmission of infectious diseases in crops and livestock, quarantined pests, invasive alien species, and living modified organisms [1]. To keep illness out of a facility, livestock producers are encouraged to maintain Biological standards in their operations. Biological (biological safety and well-being) is the set of management practices that prevent infectious diseases from being carried into a herd. The goal of a Biological program is to stop the transmission of disease-causing agents by preventing, minimizing or controlling cross-contamination of body fluids between and among animals, between animals and feed, or between animals and equipment that may directly or indirectly contact animals. Effective Biological management practices prevent the spread of disease by minimizing movement of biological organisms and their vectors onto and within the premises. These management practices are based on the principle that it is easier to prevent disease than it is to treat or react to a problem caused by disease. The outbreak of Foot and Mouth Disease in the United Kingdom in early 2001 caused many producers to reevaluate their own programs of Biological. The advantages of adopting a Biological program are numerous. An effective program can improve the cost-efficiency of the farm, improve the reputation of the producer, and allow the producer to better maintain the health status of the herd.
\end{abstract}

\section{Review Justification}

Disease Surveillance The key to success in handling Aspergillosis is early detection. If a disease can be detected in an early phase obits development, the possibility exists that it can be arrested and eliminated before it actually inflicts damage. Disease surveillance will help determine the most important diseases in farm operations and will present clinical approaches that can improve detection, diagnosis, and treatment of herd-based problems. Early detection presupposes that there is a surveillance system in place that will bring infection to light when it is first seen. Veterinarians and producers are then able to manage the problem before it becomes uncontrollable, thus protecting the local livestock industry and ensuring food security for those closely dependent upon livestock. Early detection 


\section{Discovery}

enables early warning and an early reaction. Surveillance is the primary key to effective disease management. The US livestock industry generates $\$ 100$ billion annually. Serious economic impacts are expected if Aspergillosis are introduced, suspected or prevalent. Since animals may be the primary targets of an introduced disease/epidemic, it is critical that animal populations be regularly monitored with all critical diseases reported. A systematic herd investigation strategy is pivotal to define the problems, understand important risk factors, develop a plan, and make accurate recommendations for disease management.

\section{Bioterrorism}

The study of germs offers so many connections with the diseases of animals and plants, that it certainly constitutes a first step in the ...serious investigation of putrid and contagious diseases." (Pasteur) Linkages between disease in humans and the maladies of animals continue to be a focus for those concerned with disease effects on human health. Bioterrorism is the intentional use of microorganisms or toxins derived from living organisms to cause death or disease in humans, animals, or plants on which we depend.

\section{History of Biowarfare}

In the 14thand 15thcenturies, little was known about how germs cause disease. But according to medieval medical lore, the stench of rotting bodies was known to transmit infections. During the Medieval Siege, attackers hurled dead horses and other animals by catapult (Northern France). The defenders reported that "the stink and the air were so abominable...they could not long endure" and negotiated a truce. Bodies infected with plague hurled over the walls of the city in Europe ("Black Death" in Europe). Before abandoning their attack, they sent the infected bodies of their comrades over the walls of the city. Fleeing residents carried the disease to Italy, furthering the second major epidemic of "Black Death" in Europe. During the American Revolution, British Gen. Jeffery Amherst ordered that blankets and handkerchiefs be taken from smallpox patients in the fort's infirmary and be given to Delaware Indians at a peace-making parley. During World War I, livestock were infected through needle injection and pouring bacterial cultures on animal feed. The diseases they cultivated as weapons were ganders and anthrax, both known to ravage populations of grazing animals in natural epidemics. During World War II, Japanese dropped paper bags filled with plague-infested fleas, contaminated wells and distributing poisoned foods. Cults are another avenue in which bioterrorism is instituted. In 1984, followers of the Indian guru Bagman Shree Rajneesh sprinkled Salmonella on salad bars throughout their county. The scheme was to sicken local citizens and thus prevent them from voting in an upcoming election. This attack leads to 750 cases of food poisoning. The anthrax attacks are well known for invoking more damage after the terror attacks of 


\section{Discovery}

September 11th. A week after the terrorist attacks, letters containing lethal anthrax spores were mailed. By the end of the year, 18 people had been infected with anthrax, five people had died of the inhaled form of the disease, and hundreds of millions more were struck by anxiety of the unknown. The anthrax-laced letters sparked "mass disruption" rather than "mass destruction" [4].Animal and Human Linkages There are many examples of linkages between important infectious diseases of wildlife, domestic animals, and humans such as Rift Valley Fever, Brucellosis, ad Anthrax. Each causes serious human illness that often leads to death. Animals have major roles in the ecology of each of these diseases and, like humans, also are affected by these disease agents. In the past, wildlife have been used as delivery systems for biological warfare, where these free-ranging animals were captured, infected, and released back into the wild to transmit disease to others of their kind, as well as to other susceptible species. Terrorists could use diseased wildlife to convey pathogens to wildlife and other species. The close proximity between humans and urban wildlife provides a "bridge" for the delivery of infectious disease that easily could be exploited by bioterrorists because of inadequate disease surveillance and monitoring of these wildlife. The frequent movement of pathogens through the illegal and legal transportation of wildlife attests to the need for concern regarding wildlife as Prospective vehicles for bioterrorism.

\section{Conclusion}

Biological in the context of livestock and wildlife refers to those measures taken to keep disease agents out of populations, herds, or groups of animals where they do not already exist [3]. Biological, the practice of protecting ranch and farm animals from disease, has become a major concern with the worldwide threat of Foot and Mouth Disease (FMD) and other diseases. Effective Biological requires several components including isolation, traffic control, and sanitation that aim to reduce exposure to bacteria, viruses and other organisms that may infect animals with disease. Informed veterinarians, livestock producers, and agricultural professionals are the first line of defense against foreign and other Aspergillosis. The extensive network of federal lands provides an appropriate grid for wildlife disease surveillance and monitoring to detect emerging diseases in wildlife and attendant threats for domestic animals and humans. Global efforts to combat emerging infectious disease at the wildlife-human and wildlife-domestic animal interfaces could help overcome existing deficiencies and in the end benefit national homeland security.

\section{References}

1. Koblenz Gregory D (2010) Biological Reconsidered: Calibrating Biological Threats and Responses. International Security 34(4): 96-132. 


\section{The American Journal of}

2. Mobley, R. \& Lyttle-N'guessan, C. (2009). The Herd Health Handbook for Goat Producers: Biological at the Farm Level. Florida A\&M University.

3. David A Ashford, Robyn M Kaiser, Michael E Bales, Kathleen Shut and Amie Patrawalla, teal. (2003) Planning against Biological Terrorism: Lessons from Outbreak Investigations. Emerging Infectious Diseases. 9(5).

4. NOVA Online, History of Biowarfare. Accessed April 25, 2016. 